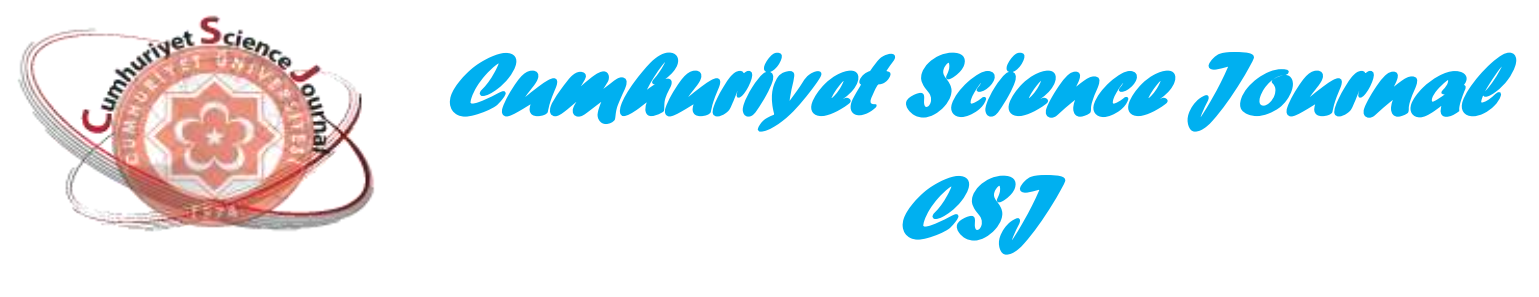

\title{
Uniqueness Theorems for Sturm-Liouville Operator with Parameter Dependent Boundary Conditions and Finite Number Of Transmission Conditions
}

\author{
Yasar CAKMAK ${ }^{1 *}$, Baki KESKIN ${ }^{I}$ \\ ${ }^{1}$ Cumhuriyet University, Faculty of Science, Department of Mathematics, 58140 Sivas, Turkey
}

Received: 03.08.2017; Accepted: 14.09.2017

http://dx.doi.org/10.17776/csj.340505

\begin{abstract}
In this paper, we prove some uniqueness theorems for the solution of inverse spectral problems of Sturm-Liouville operators with boundary conditions depending linearly on the spectral parameter and with a finite number of transmission conditions.
\end{abstract}

Keywords: Inverse problem, Sturm-Liouville operator, Weyl function, Prüfer Angle.

\section{Sonlu Sayıda Süreksizlik Koşullarına Sahip ve Sınır Koşulları \\ Parametreye Bağlı Sturm-Liouville Problemi için Teklik Teoremleri}

\begin{abstract}
Özet: Bu makalede, sonlu sayıda süreksizlik koşullarına sahip ve sınır koşulları spektral parametreye lineer şekilde bağlı Sturm-Liouville operatörlerin ters spektral problemlerinin çözümü için bazı teklik teoremleri ispatlayacağı.
\end{abstract}

Anahtar Kelimeler: Ters problem, Sturm-Liouville operatör, Weyl fonksiyonu, Prüfer Açıs1.

\section{INTRODUCTION}

First important results for inverse problem of a regular Sturm-Liouville operator were given by Ambarzumyan in 1929 [1] and Borg in 1945 [2]. In the following years, results which is obtained in these works have been generalized to various versions for Sturm-Liouville operator.

Inverse problems for Sturm-Liouville equations with boundary conditions linearly dependent on the spectral parameter were investigated in [3-14] Such problems often arise from physical problems, for example, vibration of a string, quantum mechanics and geophysics. In [7] and [12], an operator-theoretic formulation of the problems with the spectral parameter contained in only one of the boundary conditions has been given. Boundary conditions depend nonlinearly on the spectral parameter were also considered in [15-19].

Sturm-Liouville problems with transmission conditions at interior points arise in a variety of applications in applied sciences. For general background of these kind of problems, we refer (e.g.) to the monographs [21-27]. 


\section{Preliminaries:}

Consider a boundary value problem generated by the Sturm Liouville equation for $x \in I=\bigcup_{i=0}^{N}\left(d_{i}, d_{i+1}\right)$

$\ell y:=-y^{\prime \prime}+q(x) y=\lambda w(x) y$

subject to the boundary conditions

$\lambda\left(y^{\prime}(0)+h_{0} y(0)\right)=h_{1} y^{\prime}(0)+h_{2} y(0)$

$\lambda\left(y^{\prime}(1)+H_{0} y(1)\right)=H_{1} y^{\prime}(1)+H_{2} y(1)(1.3)$

and a finite number of discontinuity conditions

$$
\left\{\begin{array}{c}
y\left(d_{i}+0\right)=\alpha_{i} y\left(d_{i}-0\right) \\
y^{\prime}\left(d_{i}+0\right)=\alpha_{i}^{-1} y^{\prime}\left(d_{i}-0\right) \\
-\left(\omega_{i} \lambda+\beta_{i}\right) y\left(d_{i}-0\right)
\end{array}\right.
$$

where $q(x)$ is real valued function in $L_{2}(0,1)$; $\beta_{i}, h_{j}$ and $H_{j}, j=0,1,2$, are real numbers; $\alpha_{i} \in \mathrm{R}^{+}, \quad d_{0}=0, \quad d_{i} \in(0,1), \quad d_{N+1}=1$, $\rho_{1}:=h_{2}-h_{0} h_{1}>0, \quad \rho_{2}:=H_{0} H_{1}-H_{2}>0$, $w(x)=1 / \sigma_{k}^{2}, \quad d_{k}<x<d_{k+1}, \quad \sigma_{k} \in \mathrm{R}$ for $k=0, \ldots, N, \sigma_{0}=1$ and $\lambda$ is a spectral parameter. We denote the problem (1.1)-(1.4) by $L=L\left(q, \mathbf{h}, \mathbf{H}, \mathbf{s}_{1}, \mathbf{s}_{2}, \ldots, \mathbf{s}_{N}\right) \quad$ where $\mathbf{h}=\left(h_{0}, h_{1}, h_{2}\right), \quad \mathbf{H}=\left(H_{0}, H_{1}, H_{2}\right)$, $\mathbf{s}_{i}=\left(d_{i}, \alpha_{i}, \omega_{i}, \beta_{i}\right), i=1, \ldots, N$.

Consider a Hilbert Space $H=L_{2}(0,1) \oplus \mathrm{C}^{N+2}$, equipped with the inner product

$$
\begin{aligned}
& \langle Y, Z\rangle:=\int_{0}^{1} w^{2}(x) y(x) \overline{z(x)} d x \\
& +\sum_{i=1}^{2} \frac{1}{\rho_{i}} Y_{i} \overline{Z_{i}}+\sum_{i=1}^{N} \alpha_{i} \omega_{i} Y_{i+2} \overline{Z_{i+2}},
\end{aligned}
$$

where $\quad Y=\left(y(x) / w(x), Y_{1}, Y_{2}, \ldots, Y_{N+2}\right)^{T}$, $Z=\left(z(x) / w(x), Z_{1}, Z_{2}, \ldots, Z_{N+2}\right)^{T} \in H$.
Define an operator $T$ with the domain

$$
D(T)=\left\{Y \in H: y(x) \quad \text { and } \quad y^{\prime}(x) \quad\right. \text { are }
$$

absolutely continuous in $I, \quad \ell Y \in L_{2}(0,1)$, $y\left(d_{i}+0\right)=\alpha_{i} y\left(d_{i}-0\right), \quad Y_{1}=y^{\prime}(0)+h_{0} y(0)$, $Y_{2}=y_{1}^{\prime}(1)+H_{0} y(1), \quad Y_{i+2}=\omega_{i} y\left(d_{i}-0\right)$, $i=1, \ldots, N\}$

such that,

$T(Y)$

$$
:=\left(\begin{array}{c}
-y^{\prime \prime}(x)+q(x) y(x) \\
h_{1} y^{\prime}(0)+h_{2} y(0) \\
H_{1} y^{\prime}(1)+H_{2} y(1) \\
-y^{\prime}\left(d_{1}+0\right)+\alpha_{1}^{-1} y^{\prime}\left(d_{1}-0\right)-\beta_{1} y\left(d_{1}-0\right) \\
-y^{\prime}\left(d_{2}+0\right)+\alpha_{2}^{-1} y^{\prime}\left(d_{2}-0\right)-\beta_{2} y\left(d_{2}-0\right) \\
\cdots \\
-y^{\prime}\left(d_{N}+0\right)+\alpha_{N}^{-1} y^{\prime}\left(d_{N}-0\right)-\beta_{N} y\left(d_{N}-0\right)
\end{array}\right)
$$

It can be proven, using classical methods in the similar works (see for example [20]), that the operator $T$ is symmetric in $H$; the eigenvalues problem for the operator $T$ and the problem $L$ coincide.

Let $\varphi(x, \lambda)$ be the solution of equation (1.1) satisfying the initial conditions $y(0)=h_{1}-\lambda:=a, \quad y^{\prime}(0)=\lambda h_{0}-h_{2}:=b$ and transmission conditions (1.4). This solution is found in the interval $0=d_{0}<x<d_{1}$ by the method of the variation of parameters as

$$
\begin{aligned}
& \varphi(x, \lambda)=a \cos \sqrt{\lambda} x+\frac{b}{\sqrt{\lambda}} \sin \sqrt{\lambda} x \\
& +\frac{1}{\sqrt{\lambda}} \int_{0}^{x} \sin \sqrt{\lambda}(x-t) q(t) \varphi(t, \lambda) d t .
\end{aligned}
$$

In each interval $d_{i}<x<d_{i+1}=1$ $(i=1,2, \ldots, n)$ the solutions such as 


$$
\begin{aligned}
& \varphi(x, \lambda)=A_{i}(\sqrt{\lambda}) \cos \sqrt{\lambda} x+B_{i}(\sqrt{\lambda}) \sin \sqrt{\lambda} x \\
& +\frac{1}{\sqrt{\lambda}} \int_{0}^{x} \sin \sqrt{\lambda}(x-t) q(t) \varphi(t, \lambda) d t
\end{aligned}
$$

are searched. For each interval if the coefficients $A_{i}(\sqrt{\lambda})$ and $B_{i}(\sqrt{\lambda})$ are found by using transmission conditions (1.4) and substitued in then the following asymptotics hold for the solution $\varphi(x, \lambda)$

$$
\begin{aligned}
& \varphi(x, \lambda) \\
& =\left\{\begin{array}{l}
-\lambda \cos \sqrt{\lambda} \gamma(x) \\
+O(\sqrt{\lambda} \exp |\tau| \gamma(x)), \quad d_{0}<x<d_{1} \\
-\omega_{1} \sigma_{1} \lambda^{3 / 2} \cos \sqrt{\lambda} \gamma\left(d_{1}\right) \times \\
\times \sin \sqrt{\lambda}\left(\gamma\left(d_{1}\right)-\gamma(x)\right) \\
+O(\lambda \exp |\tau| \gamma(x)), \quad d_{1}<x<d_{2} \\
-\omega_{1} \omega_{2} \sigma_{1} \sigma_{2} \lambda^{2} \cos \sqrt{\lambda} \gamma\left(d_{1}\right) \times \\
\times \sin \sqrt{\lambda}\left(\gamma\left(d_{1}\right)-\gamma\left(d_{2}\right)\right) \times \\
\times \sin \sqrt{\lambda}\left(\gamma\left(d_{2}\right)-\gamma(x)\right) \\
+O\left(\lambda^{3 / 2} \exp |\tau| \gamma(x)\right), \quad d_{2}<x<d_{3} \\
\vdots \\
-\left(\prod_{k=1}^{n} \omega_{k} \sigma_{k}\right) \lambda^{(n+2) / 2} \cos \sqrt{\lambda} \gamma\left(d_{1}\right) \times \\
\times \sin \sqrt{\lambda}\left(\gamma\left(d_{1}\right)-\gamma\left(d_{2}\right)\right) \times \\
\times \cdots \sin \sqrt{\lambda}\left(\gamma\left(d_{n-1}\right)-\gamma\left(d_{n}\right)\right) \times \\
\times \sin \sqrt{\lambda}\left(\gamma\left(d_{n}\right)-\gamma(x)\right) \\
+O\left(\lambda^{(n+1) / 2} \exp |\tau| \gamma(x)\right), \quad d_{n}<x<d_{n+1}
\end{array}\right.
\end{aligned}
$$

$$
\begin{aligned}
& \varphi^{\prime}(x, \lambda) \\
& =\left\{\begin{array}{l}
\lambda^{312} \sin \sqrt{\lambda} \gamma(x) \\
+O(\lambda \exp |\tau| \gamma(x)), \quad d_{0}<x<d_{1} \\
\omega_{1} \lambda^{2} \cos \sqrt{\lambda} \gamma\left(d_{1}\right) \times \\
\times \cos \sqrt{\lambda}\left(\gamma\left(d_{1}\right)-\gamma(x)\right) \\
+O\left(\lambda^{312} \exp |\tau| \gamma(x)\right), \quad d_{1}<x<d_{2} \\
\omega_{1} \omega_{2} \sigma_{1} \lambda^{512} \cos \sqrt{\lambda} \gamma\left(d_{1}\right) \times \\
\times \sin \sqrt{\lambda}\left(\gamma\left(d_{1}\right)-\gamma\left(d_{2}\right)\right) \times \\
\times \cos \sqrt{\lambda}\left(\gamma\left(d_{2}\right)-\gamma(x)\right) \\
+O\left(\lambda^{2} \exp |\tau| \gamma(x)\right), \quad d_{2}<x<d_{3} \\
\vdots \\
\left(\prod_{k=1}^{n} \omega_{k} \sigma_{k-1}\right) \lambda^{(n+3) / 2} \cos \sqrt{\lambda} \gamma\left(d_{1}\right) \times \\
\times \sin \sqrt{\lambda}\left(\gamma\left(d_{1}\right)-\gamma\left(d_{2}\right)\right) \times \\
\times \cdots \sin \sqrt{\lambda}\left(\gamma\left(d_{n-1}\right)-\gamma\left(d_{n}\right)\right) \times \\
\times \cos \sqrt{\lambda}\left(\gamma\left(d_{n}\right)-\gamma(x)\right) \\
+O\left(\lambda^{(n+2) / 2} \exp |\tau| \gamma(x)\right), \quad d_{n}<x<d_{n+1}
\end{array}\right.
\end{aligned}
$$

Similarly, let $\psi(x, \lambda)$ be the solution of equation (1.1) satisfying the initial conditions $y(1)=H_{1}-\lambda:=a, \quad y^{\prime}(1)=\lambda H_{0}-H_{2}:=b$ and transmission conditions (1.4). This solution is found in the interval $d_{n}<x<d_{n+1}=1$ by the method of the variation of parameters as

$\psi(x, \lambda)=\tilde{a} \cos \sqrt{\lambda}(1-x)-\frac{\tilde{b}}{\sqrt{\lambda}} \sin \sqrt{\lambda} x$

$+\frac{1}{\sqrt{\lambda}} \int_{x}^{1} \sin \sqrt{\lambda}(t-x) q(t) \varphi(t, \lambda) d t$.

In each interval $d_{n-i}<x<d_{n-i+1}$ $(i=n, n-1, \ldots, 1)$ the solutions such as $\psi(x, \lambda)=\tilde{A}_{i}(\sqrt{\lambda}) \cos \sqrt{\lambda} x+\tilde{B}_{i}(\sqrt{\lambda}) \sin \sqrt{\lambda} x$ $+\frac{1}{\sqrt{\lambda}} \int_{0}^{x} \sin \sqrt{\lambda}(x-t) q(t) \varphi(t, \lambda) d t$

are searched. For each interval if the coefficients $\tilde{A}_{i}(\sqrt{\lambda})$ and $\tilde{B}_{i}(\sqrt{\lambda})$ are found by using transmission conditions (1.4) and written in the 
their places then the following asymptotics hold for the solution $\psi(x, \lambda)$

$\psi(x, \lambda)$

$$
=\left\{\begin{array}{l}
-\lambda \cos \sqrt{\lambda}(\gamma(1)-\gamma(x)) \\
+O(\sqrt{\lambda} \exp |\tau|(\gamma(1)-\gamma(x))), \quad d_{n}<x<d_{n+1} \\
-\omega_{n} \sigma_{n} \lambda^{3 / 2} \cos \sqrt{\lambda}\left(\gamma(1)-\gamma\left(d_{n}\right)\right) \times \\
\times \sin \sqrt{\lambda}\left(\gamma(x)-\gamma\left(d_{n}\right)\right) \\
+O(\lambda \exp |\tau|(\gamma(1)-\gamma(x))), \quad d_{n-1}<x<d_{n} \\
-\omega_{n} \omega_{n-1} \sigma_{n} \sigma_{n-1} \lambda^{2} \cos \sqrt{\lambda}\left(\gamma(1)-\gamma\left(d_{n}\right)\right) \times \\
\times \sin \sqrt{\lambda}\left(\gamma\left(d_{n-1}\right)-\gamma\left(d_{n}\right)\right) \\
\times \sin \sqrt{\lambda}\left(\gamma(x)-\gamma\left(d_{n-1}\right)\right) \\
+O\left(\lambda^{3 / 2} \exp |\tau|(\gamma(1)-\gamma(x))\right), d_{n-2}<x<d_{n-1} \\
\vdots \\
-\left(\prod_{k=1}^{n} \omega_{k} \sigma_{k}\right) \lambda^{(n+2) / 2} \cos \sqrt{\lambda}\left(\gamma(1)-\gamma\left(d_{n}\right)\right) \times \\
\times \sin \sqrt{\lambda}\left(\gamma\left(d_{n-1}\right)-\gamma\left(d_{n}\right)\right) \times \\
\times \cdots \sin \sqrt{\lambda}\left(\gamma\left(d_{1}\right)-\gamma\left(d_{2}\right)\right) \sin \sqrt{\lambda}\left(\gamma(x)-\gamma\left(d_{1}\right)\right) \\
+O\left(\lambda^{(n+1) / 2} \exp |\tau|(\gamma(1)-\gamma(x))\right), d_{0}<x<d_{1}
\end{array}\right.
$$$$
\psi^{\prime}(x, \lambda)
$$$$
\left\{\begin{array}{l}
-\lambda^{312} \sigma_{n}^{-1} \sin \sqrt{\lambda}(\gamma(1)-\gamma(x)) \\
+O(\lambda \exp |\tau|(\gamma(1)-\gamma(x))), d_{n}<x<d_{n+1} \\
-\omega_{n} \sigma_{n} \sigma_{n-1}^{-1} \lambda^{2} \cos \sqrt{\lambda}\left(\gamma(1)-\gamma\left(d_{n}\right)\right) \times \\
\times \cos \sqrt{\lambda}\left(\gamma(x)-\gamma\left(d_{n}\right)\right) \\
+O\left(\lambda^{312} \exp |\tau|(\gamma(1)-\gamma(x))\right), d_{n-1}<x<d_{n} \\
-\omega_{n} \omega_{n-1} \sigma_{n} \sigma_{n-1} \sigma_{n-2}^{-1} \lambda^{512} \cos \sqrt{\lambda}\left(\gamma(1)-\gamma\left(d_{n}\right)\right) \times
\end{array}\right.
$$$$
=\left\{\begin{array}{l}
\times \sin \sqrt{\lambda}\left(\gamma\left(d_{n-1}\right)-\gamma\left(d_{n}\right)\right) \\
\times \cos \sqrt{\lambda}\left(\gamma(x)-\gamma\left(d_{n-1}\right)\right) \\
+O\left(\lambda^{2} \exp |\tau|(\gamma(1)-\gamma(x))\right), d_{n-2}<x<d_{n-1}
\end{array}\right.
$$$$
-\left(\prod_{k=1}^{n} \omega_{k} \sigma_{k}\right) \lambda^{(n+3) / 2} \cos \sqrt{\lambda}\left(\gamma(1)-\gamma\left(d_{n}\right)\right) \times
$$$$
\times \sin \sqrt{\lambda}\left(\gamma\left(d_{n-1}\right)-\gamma\left(d_{n}\right)\right) \times
$$$$
\times \cdots \sin \sqrt{\lambda}\left(\gamma\left(d_{1}\right)-\gamma\left(d_{2}\right)\right) \times
$$$$
\times \cos \sqrt{\lambda}\left(\gamma(x)-\gamma\left(d_{1}\right)\right)
$$$$
+O\left(\lambda^{(n+2) / 2} \exp |\tau|(\gamma(1)-\gamma(x))\right), d_{0}<x<d_{1}
$$

These solutions are entire functions of $\lambda$ and satisfy the relation $\psi\left(x, \lambda_{n}\right)=\beta_{n} \varphi\left(x, \lambda_{n}\right)$ for each eigenvalue $\lambda_{n}$, where $\beta_{n}=-\frac{\psi^{\prime}\left(0, \lambda_{n}\right)+h_{0} \psi\left(0, \lambda_{n}\right)}{\rho_{1}}$.

The characteristic function $\Delta(\lambda)$ and norming constants $\alpha_{n}$ of the problem $L$ are defined as follows.

$\Delta(\lambda)=W[\varphi, \psi]$

$=\lambda\left(\varphi^{\prime}(1, \lambda)+H_{0} \varphi(1, \lambda)\right)$

$-H_{1} \varphi^{\prime}(1, \lambda)-H_{2} \varphi(1, \lambda)$

$=-\lambda\left(\psi^{\prime}(0, \lambda)+h_{0} \psi(0, \lambda)\right)$

$+h_{1} \psi^{\prime}(0, \lambda)+h_{2} \psi(0, \lambda)$

$\alpha_{n}:=\int_{0}^{1} w(x) \varphi^{2}\left(x, \lambda_{n}\right) d x$

$+\frac{1}{\rho_{1}}\left(\varphi^{\prime}\left(0, \lambda_{n}\right)+h_{0} \varphi\left(0, \lambda_{n}\right)\right)^{2}$

$+\frac{1}{\rho_{2}}\left(\varphi^{\prime}\left(1, \lambda_{n}\right)+H_{0} \varphi\left(1, \lambda_{n}\right)\right)^{2}$

$+\sum_{i=1}^{N} \alpha_{i} \omega_{i} \varphi^{2}\left(d_{i}-0, \lambda_{n}\right)$

It is obvious that, $\Delta(\lambda)$ is an entire function in $\lambda$ and the zeros namely $\left\{\lambda_{n}\right\}$ of $\Delta(\lambda)$ coincide with eigenvalues of the problem $L$. Now, from the asymptotics of solutions $\varphi(x, \lambda)$ and $\psi(x, \lambda)$, we can write

$$
\begin{aligned}
& \Delta(\lambda)=\lambda^{(N+5) / 2}\left(\prod_{i=1}^{N} \omega_{i} \sigma_{i-1}\right) \times \\
& \times \cos \sqrt{\lambda} \gamma\left(d_{1}\right) \times \\
& \times \sin \sqrt{\lambda}\left(\gamma\left(d_{1}\right)-\gamma\left(d_{2}\right)\right) \times \\
& \times \ldots \sin \sqrt{\lambda}\left(\gamma\left(d_{N-1}\right)-\gamma\left(d_{N}\right)\right) \times \\
& \times \cos \sqrt{\lambda}\left(\gamma\left(d_{N}\right)-\gamma(1)\right) \\
& +O\left(\lambda^{(N+4) / 2} \exp |\tau| \gamma(1)\right)
\end{aligned}
$$


where $\tau=\operatorname{Im} \sqrt{\lambda}, \gamma(x)=\int_{0}^{x} \sqrt{w(t)} d t$

Lemma $1 i$-All eigenvalues of the problem $L$ are real.

ii- $\Delta^{\prime}\left(\lambda_{n}\right)=\beta_{n} \alpha_{n}$, so eigenvalues are simple zeros of $\Delta(\lambda)$.

iii- Two eigenfunctions $\varphi\left(x, \lambda_{1}\right)$ and $\varphi\left(x, \lambda_{2}\right)$, corresponding to different eigenvalues $\lambda_{1}$ and $\lambda_{2}$, are orthogonal, i.e.,

$$
\begin{aligned}
& \int_{0}^{1} \varphi\left(x, \lambda_{1}\right) \varphi\left(x, \lambda_{2}\right) d x \\
& +\frac{1}{\rho_{1}}\left(\varphi^{\prime}\left(0, \lambda_{1}\right)+h_{0} \varphi\left(0, \lambda_{1}\right)\right) \times \\
& \times\left(\varphi^{\prime}\left(0, \lambda_{2}\right)+h_{0} \varphi\left(0, \lambda_{2}\right)\right) \\
& +\frac{1}{\rho_{2}}\left(\varphi^{\prime}\left(1, \lambda_{1}\right)+H_{0} \varphi\left(1, \lambda_{1}\right)\right) \times \\
& \times\left(\varphi^{\prime}\left(1, \lambda_{2}\right)+H_{0} \varphi\left(1, \lambda_{2}\right)\right) \\
& +\sum_{i=1}^{N} \alpha_{i} \omega_{i} \varphi\left(d_{i}-0, \lambda_{1}\right) \varphi\left(d_{i}-0, \lambda_{2}\right)=0 .
\end{aligned}
$$

Proof. Since the operator $T$ is selfadjoint, all eigenvalues are real and two different eigenfunctions are orthogonal. This proves (i) and (iii). Let us show that the simplicity of the eigenvalues $\lambda_{n}$; write the following equations,

$$
\begin{aligned}
& \psi^{\prime \prime}(x, \lambda)+q(x) \psi(x, \lambda)=\lambda w(x) \psi(x, \lambda), \\
& \varphi^{\prime \prime}\left(x, \lambda_{n}\right)+q(x) \varphi\left(x, \lambda_{n}\right)=\lambda_{n} w(x) \varphi\left(x, \lambda_{n}\right) .
\end{aligned}
$$

If these equations are multiplied by $\varphi\left(x, \lambda_{n}\right)$ and $\psi(x, \lambda)$, respectively and subtracting them side by side and finally integrating over the interval $[0,1]$, the equality

$$
\begin{aligned}
& \left.\left[\varphi^{\prime}\left(x, \lambda_{n}\right) \psi(x, \lambda)-\psi^{\prime}(x, \lambda) \varphi\left(x, \lambda_{n}\right)\right]\right]_{0}^{d_{1}-0} \\
& +\left[\varphi^{\prime}\left(x, \lambda_{n}\right) \psi(x, \lambda)-\psi^{\prime}(x, \lambda) \varphi\left(x, \lambda_{n}\right)\right]_{d_{1}+0}^{d_{2}-0} \\
& +\ldots+ \\
& +\left[\varphi^{\prime}\left(x, \lambda_{n}\right) \psi(x, \lambda)-\psi^{\prime}(x, \lambda) \varphi\left(x, \lambda_{n}\right)\right]_{d_{N}+0}^{1} \\
& =\left(\lambda-\lambda_{n}\right) \int_{0}^{1} w(x) \psi(x, \lambda) \varphi\left(x, \lambda_{n}\right) d x
\end{aligned}
$$

is obtained. Add and subtract $\Delta(\lambda)$ in the lefthand side of the last equality and use initial conditions (1.5) to get

$$
\begin{aligned}
& \Delta(\lambda)+\left(\lambda-\lambda_{n}\right)\left(\psi^{\prime}(0, \lambda)+h_{0} \psi(0, \lambda)\right) \\
& -\left(\lambda-\lambda_{n}\right)\left(\varphi^{\prime}\left(1, \lambda_{n}\right)+H_{0} \varphi\left(1, \lambda_{n}\right)\right) \\
& -\left(\lambda-\lambda_{n}\right) \sum_{i=1}^{N} \alpha_{i} \gamma_{i} \psi\left(d_{i}-0, \lambda\right) \varphi\left(d_{i}-0, \lambda_{n}\right) \\
& =\left(\lambda-\lambda_{n}\right) \int_{0}^{1} w(x) \psi(x, \lambda) \varphi\left(x, \lambda_{n}\right) d x
\end{aligned}
$$

rewrite this equality as

$$
\begin{aligned}
& \frac{\Delta(\lambda)}{\lambda-\lambda_{n}}=\int_{0}^{1} w(x) \psi(x, \lambda) \varphi\left(x, \lambda_{n}\right) d x \\
& +\left(\varphi^{\prime}\left(1, \lambda_{n}\right)+H_{0} \varphi\left(1, \lambda_{n}\right)\right) \\
& -\left(\psi^{\prime}(0, \lambda)+h_{0} \psi(0, \lambda)\right) \\
& +\sum_{i=1}^{N} \alpha_{i} \gamma_{i} \psi\left(d_{i}-0, \lambda\right) \varphi\left(d_{i}-0, \lambda_{n}\right)
\end{aligned}
$$$$
=\int_{0}^{1} w(x) \psi(x, \lambda) \varphi\left(x, \lambda_{n}\right) d x
$$$$
-\frac{\left(\psi^{\prime}(0, \lambda)+h_{0} \psi(0, \lambda)\right)\left(\varphi^{\prime}\left(0, \lambda_{n}\right)+h_{0} \varphi\left(0, \lambda_{n}\right)\right)}{h_{0} h_{1}-h_{2}}
$$$$
+\frac{\left(\varphi^{\prime}\left(1, \lambda_{n}\right)+H_{0} \varphi\left(1, \lambda_{n}\right)\right)\left(\psi^{\prime}(1, \lambda)+H_{0} \psi(1, \lambda)\right)}{H_{0} H_{1}-H_{2}}
$$$$
+\sum_{i=1}^{N} \alpha_{i} \gamma_{i} \psi\left(d_{i}-0, \lambda\right) \varphi\left(d_{i}-0, \lambda_{n}\right)
$$$$
=\int_{0}^{1} w(x) \psi(x, \lambda) \varphi\left(x, \lambda_{n}\right) d x
$$$$
+\frac{\left(\psi^{\prime}(0, \lambda)+h_{0} \psi(0, \lambda)\right)\left(\varphi^{\prime}\left(0, \lambda_{n}\right)+h_{0} \varphi\left(0, \lambda_{n}\right)\right)}{\rho_{1}}
$$ 
$+\frac{\left(\varphi^{\prime}\left(1, \lambda_{n}\right)+H_{0} \varphi\left(1, \lambda_{n}\right)\right)\left(\psi^{\prime}(1, \lambda)+H_{0} \psi(1, \lambda)\right)}{\rho_{2}}$

$+\sum_{i=1}^{N} \alpha_{i} \gamma_{i} \psi\left(d_{i}-0, \lambda\right) \varphi\left(d_{i}-0, \lambda_{n}\right)$

As $\lambda \rightarrow \lambda_{n}$ and from the equalities $\psi\left(x, \lambda_{n}\right)=\beta_{n} \varphi\left(x, \lambda_{n}\right)$ and $(1.7)$

$$
\Delta^{\prime}\left(\lambda_{n}\right)=\beta_{n} \alpha_{n}
$$

is obtained. Thus $\Delta^{\prime}\left(\lambda_{n}\right) \neq 0$.

\section{Main Results:}

In this section, we prove three theorems, uniquely determined by i) Weyl function, ii) Prüfer angle and iii) eigenvalues and norming constants, for uniqueness of the solution of the inverse problem. We consider a boundary value problem $\tilde{L}$, together with $L$, of the same form but with different coefficients $\tilde{q}(x), \tilde{\mathbf{h}}, \tilde{\mathbf{H}}, \widetilde{\mathbf{s}}_{i}$, $i=1, \ldots, N$.

Let the function $\chi(x, \lambda)$ denotes the solution of (1.1) under the initial conditions $\chi(0, \lambda)=\rho_{1}^{-1}, \quad \chi^{\prime}(0, \lambda)=-\rho_{1}^{-1} h_{0}$ and the transmission conditions (1.4). It is clear that the solution $\psi(x, \lambda)$ satisfies the following relation

$$
\begin{aligned}
& \psi(x, \lambda)=\Delta(\lambda) \chi(x, \lambda) \\
& -\frac{\psi^{\prime}(0, \lambda)+h_{0} \psi(0, \lambda)}{\rho_{1}} \varphi(x, \lambda)
\end{aligned}
$$

If we denote

$$
m(\lambda):=-\frac{\psi^{\prime}(0, \lambda)+h_{0} \psi(0, \lambda)}{\rho_{1} \Delta(\lambda)}
$$

then we have

$$
\frac{\psi(x, \lambda)}{\Delta(\lambda)}=\chi(x, \lambda)+m(\lambda) \varphi(x, \lambda) .
$$

The function $m(\lambda)$ is called the Weyl function of the boundary value problem (1.1)(1.4).

Theorem 1 If $m(\lambda)=\tilde{m}(\lambda)$, then $L=\tilde{L}$, i.e., $q(x)=\tilde{q}(x)$, almost everywhere in $I ; \mathbf{h}=\tilde{\mathbf{h}}$, $\mathbf{H}=\tilde{\mathbf{H}}$ and $\mathbf{s}_{i}=\widetilde{\mathbf{s}}_{i}, i=1, \ldots, n$.

Proof. Let us define the functions $P_{1}(x, \lambda)$ and $P_{2}(x, \lambda)$ as follows,

$P_{1}(x, \lambda)$

$=\varphi(x, \lambda) \tilde{\Phi}^{\prime}(x, \lambda)-\Phi(x, \lambda) \tilde{\varphi}^{\prime}(x, \lambda)$

$P_{2}(x, \lambda)$

$=\Phi(x, \lambda) \tilde{\varphi}(x, \lambda)-\varphi(x, \lambda) \tilde{\Phi}(x, \lambda)$

where $\Phi(x, \lambda)=\frac{\psi(x, \lambda)}{\Delta(\lambda)}$. If $m(\lambda)=\tilde{m}(\lambda)$ then from (2.3)-(2.5), $P_{1}(x, \lambda)$ and $P_{2}(x, \lambda)$ are entire functions in $\lambda$. Denote $G_{\delta}=\left\{\lambda \in \mathrm{C}: \lambda=k^{2},\left|k-k_{n}\right|>\delta, n=1,2, \ldots\right\}$ and

$\tilde{G}_{\delta}=\left\{\lambda \in \mathrm{C}: \lambda=k^{2},\left|k-\tilde{k}_{n}\right|>\delta, n=1,2, \ldots\right\}$, where $\delta$ is sufficiently small number, $k_{n}$ and $\tilde{k}_{n}$ are square roots of the eigenvalues of the problem $L$ and $\tilde{L}$, respectively. One can easily show that the asymptotics for $d_{i}<x<d_{i+1}, i=0, \ldots, n$

$\left\{\begin{array}{l}\Phi(x, \lambda)=O\left(\lambda^{-\frac{i+3}{2}} \exp (-|\tau| \gamma(x))\right) \\ \Phi^{\prime}(x, \lambda)=O\left(\lambda^{-\frac{i+2}{2}} \exp (-|\tau| \gamma(x))\right)\end{array}\right.$

are valid for sufficiently large $|\lambda|$. Thus, the following inequalities are obtained from the asymptotics

$$
\begin{aligned}
& \left|P_{1}(x, \lambda)\right| \leq C_{\delta}, \\
& \left|P_{2}(x, \lambda)\right| \leq C_{\delta}|\lambda|^{-1 / 2}, \lambda \in G_{\delta} \cap \tilde{G}_{\delta}
\end{aligned}
$$


According to the last inequalities and Liouville's theorem, $P_{1}(x, \lambda)=A(x)$ and $P_{2}(x, \lambda) \equiv 0, \quad$ for $\quad x \in[0,1][1$ $\left\{a_{1}, d_{2}, \tilde{d}_{1}, \tilde{d}_{2}, \ldots, \tilde{d}_{n-1}, \tilde{d}_{n}\right\}$. Use (2.4) and (2.5) again to take

$$
\begin{aligned}
& \varphi(x, \lambda)=A(x) \tilde{\varphi}(x, \lambda), \\
& \Phi(x, \lambda)=A(x) \tilde{\Phi}(x, \lambda) .
\end{aligned}
$$

Since $W[\Phi(x, \lambda), \varphi(x, \lambda)] \equiv 1$ and similarly $W[\tilde{\Phi}(x, \lambda), \tilde{\varphi}(x, \lambda)] \equiv 1$, then $A^{2}(x)=1$ for $x$ in $I$.

On the other hand, the asymptotic expressions

$$
\begin{aligned}
& \varphi(x, \lambda) \\
& =\theta(\lambda) \exp (-i \sqrt{\lambda} \gamma(x))(1+o(1)) \\
& \tilde{\varphi}(x, \lambda) \\
& =\tilde{\theta}(\lambda) \exp (-i \sqrt{\lambda} \gamma(x))(1+o(1))
\end{aligned}
$$

is valid for sufficiently large $\lambda$ on the imaginary axis, where

$$
\theta(\lambda)=\left\{\begin{array}{l}
\frac{\lambda}{2}, d_{0}<x<d_{1} \\
-\frac{1}{2}\left(\prod_{k=1}^{i} \omega_{k} \sigma_{k}\right) \lambda^{(i+2) / 2} C_{i}, d_{i}<x<d_{i+1}
\end{array}\right.
$$

$$
\tilde{\theta}(\lambda)=\left\{\begin{array}{l}
\frac{\lambda}{2}, \tilde{d}_{0}<x<\tilde{d} \\
-\frac{1}{2}\left(\prod_{k=1}^{i} \tilde{\omega}_{k} \sigma_{k}\right) \lambda^{(i+2) / 2} \tilde{C}_{i}, \tilde{d}_{i}<x<\tilde{d}_{i+1}
\end{array}\right.
$$

$$
C_{i}=\cos \sqrt{\lambda} \gamma\left(d_{1}\right) \prod_{i=1}^{n} \sin \sqrt{\lambda}\left(\gamma\left(d_{i-1}\right)-\gamma\left(d_{i}\right)\right),
$$$$
\tilde{C}_{i}=\cos \sqrt{\lambda} \gamma\left(\tilde{d}_{1}\right) \prod_{i=1}^{n} \sin \sqrt{\lambda}\left(\gamma\left(\tilde{d}_{i-1}\right)-\gamma\left(\tilde{d}_{i}\right)\right),
$$
$i=1, \ldots, n, \quad d_{0}=0, \quad d_{n+1}=1, \quad \tilde{d}_{0}=0 \quad$ and $\tilde{d}_{n+1}=1$.
From (2.8)-(2.12), we can see that $d_{i}=\tilde{d}_{i}$ for $i=\overline{1, n}$. Moreover, if we use $\theta(\lambda)=A(x) \tilde{\theta}(\lambda)$ and $A^{2}(x)=1$, we get $\omega_{i}=\tilde{\omega}_{i}$ for $i=1, \ldots, n$ and $A(x)=1$ from (2.11)-(2.12). Hence,

$\varphi(x, \lambda) \equiv \tilde{\varphi}(x, \lambda)$ and $\frac{\psi^{\prime}(x, \lambda)}{\psi(x, \lambda)} \equiv \frac{\tilde{\psi}^{\prime}(x, \lambda)}{\tilde{\psi}(x, \lambda)}$

It can be obtained from (1.1)-(1.4) that $q(x)=\tilde{q}(x)$, a.e. in $I ; \mathbf{s}_{i}=\widetilde{\mathbf{s}}_{i}, \quad i=1, \ldots, n$ and $\mathbf{h}=\tilde{\mathbf{h}}, \mathbf{H}=\tilde{\mathbf{H}}$. Consequently $L=\tilde{L}$.

The function called Prüfer angle is defined by

$$
\begin{aligned}
& P(\lambda) \\
& := \begin{cases}\cot ^{-1} \frac{\psi^{\prime}(0, \lambda)}{\psi(0, \lambda)} & \text { if } \psi(0, \lambda) \neq 0, \\
\tan ^{-1} \frac{\psi(0, \lambda)}{\psi^{\prime}(0, \lambda)} & \text { if } \psi^{\prime}(0, \lambda) \neq 0\end{cases}
\end{aligned}
$$

Theorem 2 If $P(\lambda)=\tilde{P}(\lambda)$ and $\mathbf{h}=\tilde{\mathbf{h}}$, $L=\tilde{L}$; i.e. the problem $L$ is uniquely determined by $P(\lambda)$ and $U(y)$.

Proof. It is obvious from (6), (11) and (22) that the equality

$$
m(\lambda)\left[\lambda-\frac{h_{1} \cot P(\lambda)+h_{2}}{h_{0}+\cot P(\lambda)}\right]=-\rho_{1}^{-1}
$$

holds. Therefore, under the hypothesis of the theorem, we get $m(\lambda)=\tilde{m}(\lambda)$. This completes the proof.

Theorem 3 The problem $L$ is uniquely determined by $\left\{\lambda_{n}, \alpha_{n}\right\}_{n \geq 0}$

Proof. The meromorphic function $m(\lambda)$ has simple poles at $\lambda_{n}$ and its residues at these poles are 


$$
\begin{aligned}
& \operatorname{Res}\left\{m(\lambda), \lambda_{n}\right\}=\frac{\psi^{\prime}\left(0, \lambda_{n}\right)+h_{0} \psi\left(0, \lambda_{n}\right)}{\rho_{1} \Delta^{\prime}\left(\lambda_{n}\right)} \\
& =-\frac{\beta_{n}}{\Delta^{\prime}\left(\lambda_{n}\right)}=-\frac{1}{\alpha_{n}} .
\end{aligned}
$$

Denote $\Gamma_{n}=\left\{\lambda: \lambda=k^{2}, \quad|k|=\sqrt{\lambda_{n}}+\varepsilon\right\}$, where $\varepsilon$ is sufficiently small number. Consider the contour integral

$$
F_{n}(\lambda)=\frac{1}{2 \pi i} \int_{\Gamma_{n}} \frac{m(\eta)}{(\eta-\lambda)} d \eta, \lambda \in \operatorname{int} \Gamma_{n} .
$$

It can be calculated that $\lim _{n \rightarrow \infty} F_{n}(\lambda)=0$ and from Residue theorem that

$$
m(\lambda)=\sum_{n=0}^{\infty} \frac{1}{\alpha_{n}\left(\lambda_{n}-\lambda\right)}
$$

Consequently, if $\lambda_{n}=\tilde{\lambda}_{n}$ and $\alpha_{n}=\tilde{\alpha}_{n}$ for all $n$, then from (2.14), $m(\lambda)=\tilde{m}(\lambda)$. Hence, Theorem 1 yields $L=\tilde{L} \quad$ when $\left\{\lambda_{n}, \alpha_{n}\right\}_{n \geq 0}=\left\{\tilde{\lambda}_{n}, \tilde{\alpha}_{n}\right\}_{n \geq 0}$.

Acknowledgments: The first author is supported by the Scientific Research Project Fund of Cumhuriyet University under the project number F-442.

\section{REFERENCES}

[1]. Ambartsumyan, V. A., Uber eine frage der eigenwerttheorie, Zeitschrift für Physik, 1929, 53, 690-695.

[2]. Borg, G., Eine umkehrung der SturmLiouvilleschen eigenwertaufgabe. Bestimmung der differentialgleichung durch die eigenwerte, Acta Math., 1946, 78, 1-96.

[3]. Benedek, A. and Panzone, R., On inverse eigenvalue problems for a second-order differential equations with parameter contained in the boundary conditions, Notas de Algebra y Analisis, 1980, 9, 113.

[4]. Binding, P. A., Browne, P. J. and Watson, B. A., Inverse spectral problems for Sturm-Liouville equations with eigenparameter dependent boundary conditions, J. London Math. Soc., 2000, 62, 161-182.

[5]. Browne, P. J. and Sleeman, B. D., A uniqueness theorem for inverse eigenparameter dependent SturmLiouville problems, Inverse Problems, 1997, 13, 1453-1462.

[6]. Chugunova, M. V., Inverse spectral problem for the Sturm-Liouville operator with eigenvalue parameter dependent boundary conditions, Oper. Theory: Adv. Appl., 2001, 123 (Basel: Birkhauser), 18794.

[7]. Fulton, C. T., Two-point boundary value problems with eigenvalue parameter contained in the boundary conditions, Proc. R. Soc. Edinburgh, 1977, A77, 293-308.

[8]. Fulton, C. T., Singular eigenvalue problems with eigenvalue parameter contained in the boundary conditions, Proc. R. Soc. Edinburgh, 1980, A87, 1-34.

[9]. Keskin, B., Ozkan, A. S. and Yalçın, N., Inverse spectral problems for discontinuous Sturm-Liouville operator with eigenparameter dependent boundary conditions, Commun. Fac. Sci. Univ. Ank. Series A1, 2011, 60(1), 1, 15-25.

[10].Ozkan, A. S. and Keskin, B., Spectral problems for Sturm-Liouville operator with boundary and jump conditions linearly dependent on the eigenparameter, Inverse Problems in Science and Engineering, 2012, 20(6), 799-808.

[11].Ozkan, A. S., Keskin, B. and Cakmak, Y., Double Discontinuous Inverse Problems for Sturm-Liouville Operator with Parameter-Dependent Conditions, Abstract and Applied Analysis, 2013, Article ID 794262, p.7.

[12].Walter, J., Regular eigenvalue problems with eigenvalue parameter in the boundary condition, Math. Z., 1973, 133, 301-312.

[13].Wang, Y. P., Inverse problems for SturmLiouville operators with interior discontinuities and boundary conditions dependent on the spectral parameter, Mathematical Methods in the Applied Sciences, 2013, 36(7), 857-868.

[14].Yang, C. F. and Huang, Z. Y., A halfinverse problem with eigenparameter 
dependent boundary conditions, Numerical Functional Analysis and Optimization, 2010, 31(6), 754-762.

[15].Binding, P. A., Browne, P. J. and Seddighi, K., Sturm-Liouville problems with eigenparameter dependent boundary conditions, Proc. Edinburgh Math. Soc.,1993, 2(37), 57-72.

[16].Binding, P. A. and Browne, P. J., Oscillation theory for indefinite SturmLiouville problems with eigenparameterdependent boundary conditions, Proc. $R$. Soc. Edinburgh A, 1997, 127, 1123-1136.

[17].Binding, P. A., Browne, P. J. and Watson, B. A., Equivalence of inverse SturmLiouville problems with boundary conditions rationally dependent on the eigenparameter, J. Math. Anal. Appl., 2004, 291, 246-261.

[18].Mennicken, R., Schmid, H. and Shkalikov, A. A., On the eigenvalue accumulation of Sturm-Liouville problems depending nonlinearly on the spectral parameter, Math. Nachr., 1998, 189, 157-170.

[19].Schmid, H. and Tretter, C., Singular Dirac systems and Sturm-Liouville problems nonlinear in the spectral parameter, Journal of Differential Equations, 2002, 181(2), 511-542.

[20].Altinisik, N., Kadakal, M. and Mukhtarov, O. Sh., Eigenvalues and eigenfunctions of discontinuous Sturm-Liouville problems with eigenparameter dependent boundary conditions, Acta Math. Hungar., 2004, 102(1-2), 159-175.

[21].Amirov, R. Kh., Ozkan, A. S. and Keskin, B., Inverse problems for impulsive SturmLiouville operator with spectral parameter linearly contained in boundary conditions, Integral Transforms and Special Functions, 2009, 20(8), 607-618.

[22].Freiling, G. and Yurko, V., Inverse SturmLiouville problems and their applications, Nova Science, New York, 2001.

[23].Hald, O. H., Discontinuous inverse eigenvalue problems, Comm. Pure Appl. Math., 1984, 37, 539-577.

[24].Ozkan, A. S., Inverse Sturm-Liouville problems with eigenvalue dependent boundary and discontinuity conditions, Inverse Probl. Sci. Eng., 2012, 20(6), 857868.

[25].Yurko, V. A., Integral transforms connected with discontinuous boundary value problems, Integral Transforms Spec. Funct., 2000, 10, 141-164.

[26].Yurko ,V. A., Method of spectral mappings in the inverse problem theory, Inverse Illposed Problems Ser., VSP, Utrecht, 2002.

[27].Guldu, Y., Inverse Eigenvalue Problems for a Discontinuous Sturm-Liouville Operator with Two Discontinuities, Boundary Value Problems, 2013, DOI:10.1186/1687-2770-2013-209. 